\title{
Disponibilidade e lixiviação de nitrogênio em dois solos típicos de Pontes e Lacerda, MT, tratados com resíduo ruminal bovino
}

\section{Availability and leaching of nitrogen in two typical soils of Pontes e Lacerda, MT, treated with bovine rumen residue}

\author{
Fernando Luiz Silva ${ }^{{ }^{*}}$; Maria Aparecida Pereira Pierangeli; \\ Raquel Joana Trautmann-Machado ${ }^{3}$
}

\begin{abstract}
Resumo
A alta produção de resíduos durante o abate de bovinos aumenta a necessidade de promover a sua correta utilização. Neste estudo, a disponibilidade e a lixiviação de $\mathrm{N}\left(\mathrm{NO}_{3}{ }^{-} \mathrm{e} \mathrm{H}_{4}^{+}\right)$, após a incorporação resíduo ruminal bovino (RRB), foi avaliada em dois solos típicos de Pontes e Lacerda, MT: um Latossolo Vermelho distrófico e um Argissolo Vermelho-Amarelo eutrófico. O experimento foi conduzido em casa de vegetação da Universidade do Estado de Mato Grosso, UNEMAT, entre dezembro de 2009 e março de 2010. Foram coletadas amostras indeformadas em tubos de PVC (40 cm de comprimento; 10 $\mathrm{cm}$ de diâmetro), nos quais as doses de RRB foram adicionados para proporcionar 0, 50, 100, e 400 $\mathrm{kg}$ de $\mathrm{N} \mathrm{ha}^{-1}$ e adicionou-se ureia para proporcionar $400 \mathrm{~kg} \mathrm{~N} \mathrm{ha}^{-1}$. Cada tratamento foi realizado com 4 repetições. As colunas foram irrigados semanalmente, e o lixiviado foi recolhido e armazenado para análise. Após o experimento, amostras de solo foram coletados de $10 \mathrm{em} 10 \mathrm{~cm}$ e as concentrações de $\mathrm{NO}_{3}-\mathrm{e} \mathrm{NH}_{4}^{+}$foram determinadas. $\mathrm{O}$ uso da $\mathrm{RRB}$ resultou em maior disponibilidade de $\mathrm{N}$ no Latossolo Vermelho distrófico, enquanto no Argissolo Vermelho-Amarelo eutrófico o RRB não pode fornecer uma quantidade adequada de nitrogênio ao solo no período estudado. Em termos de lixiviação, o Latossolo Vermelho distrófico, tem maior capacidade de retenção de $\mathrm{NO}_{3}^{-}$e menor capacidade de retenção de $\mathrm{NH}_{4}{ }^{+}$do que o Argissolo Vermelho-Amarelo eutrófico, no entanto, os níveis de $\mathrm{NO}_{3}^{-} \mathrm{e} \mathrm{NH}_{4}^{+}$no lixiviado coletado dos dois solos estavam dentro dos valores normais estabelecidos pela Resolução Conama 357/2005. O uso de RRB, em comparação com a utilização de ureia resultou num menor risco de lixiviação $\mathrm{N}$.

Palavras-chave: Argissolo, Latossolo, nitrato, resíduo orgânico
\end{abstract}

\begin{abstract}
High production of waste during the slaughter of cattle increases the need to promote their correct utilization. In this study, the availability and leaching of nitrogen $\left(\mathrm{NO}_{3}^{-}\right.$and $\left.\mathrm{NH}_{4}^{+}\right)$after bovine rumen residue (BRR) incorporation was evaluated in 2 typical soils of Pontes e Lacerda, MT: a distrophic Red Latosol and a eutrophic Yellow-Red Argisol. The experiment was conducted in a greenhouse at the University of State of Mato Grosso, UNEMAT, between December 2009 and March 2010. The samples were undisturbed soil in PVC tubes (length $40 \mathrm{~cm}$; diameter, $10 \mathrm{~cm}$ ), in which doses of BRR were added to provide $0,50,100$, and $400 \mathrm{~kg} \mathrm{~N} \mathrm{ha}^{-1}$ and urea was added to provide $400 \mathrm{~kg} \mathrm{~N} \mathrm{ha}^{-1}$. Each treatment
\end{abstract}

\footnotetext{
${ }^{1}$ Zootecnista, Discente do Programa de Pós-graduação em Ciências Ambientais, Universidade do Estado de Mato Grosso, UNEMAT, campus de Cáceres, MT. E-mail: fernandoluiz_s@hotmail.com

${ }^{2}$ Eng $^{\mathrm{a}} \mathrm{Agr}^{\mathrm{a}}$, Dr ${ }^{\mathrm{a}}$ em Solos e Nutrição de Plantas. Prof ${ }^{\mathrm{a}}$. Adjunta do Dept ${ }^{\mathrm{o}}$ de Zootecnia, UNEMAT, Campus de Pontes e Lacerda, MT. E-mail: mapp@unemat.br

${ }^{3}$ Zootecnista, M.e. em Ciências Ambientais, Prof ${ }^{a}$. Associada do Dept ${ }^{\circ}$ de Zootecnia, UNEMAT, Campus de Pontes e Lacerda, MT. E-mail: raquel trautmann@hotmail.com

* Autor para correspondência
} 
was performed using 4 replicates. The columns were irrigated weekly, and the leachate was collected and stored for analysis. After the experiment, soil samples were collected from 10 to $10 \mathrm{~cm}$ and the $\mathrm{NO}_{3}^{-}$ and $\mathrm{NH}_{4}^{+}$concentrations were determined. The use of BRR resulted in higher potential availability of $\mathrm{N}$ in distrophic Red Latosol, while in the eutrophic Yellow-Red Argisol, BRR could not supply adequate amount of nitrogen to the soil within the study period. In terms of $\mathrm{N}$ leaching, the distrophic Red Latosol has higher retention capacity for $\mathrm{NO}_{3}^{-}$and lower retention capacity for $\mathrm{NH}_{4}^{+}$than eutrophic Yellow-Red Argisol; however, the levels of $\mathrm{NO}_{3}{ }^{-}$and $\mathrm{NH}_{4}{ }^{+}$in the leachate collected from the 2 soils were within the standard values established by Resolution Conama 357/2005. The use of BRR as compared to the use of urea resulted in a lower risk of leaching $\mathrm{N}$.

Ke ywords: Argisol, Latosol, nitrate, organic waste

\section{Introdução}

A destinação correta de resíduos urbanos, agrícolas e industriais, tais como lodo de esgoto e resíduos de produção animal, tem sido uma problemática abordada por muitos pesquisadores, devido aos riscos de contaminação dos recursos naturais por excesso de alguns elementos que podem ter efeitos tóxicos, tais como metais pesados e nitrogênio, ou mesmo pela desoxigenação de corpos d'água, presença de microrganismos patogênicos e produção de odor desagradável (BETTIOL; FERNANDES, 2004; JULIATTI et al., 2002; MIYAZAWA; BARBOSA; PARRA, 2009).

A agricultura brasileira vem avançando no cenário mundial, conquistando liderança na produção e exportação de diversos produtos agrícolas, assumindo papel importante também no seguimento de agroenergia. Neste cenário, a importância da adubação, principalmente nitrogenada tem se destacado com relação à produção de alimentos e de biomassa energética. Considerando que a expansão de áreas agricultáveis está tornando-se limitada e as necessidades de preservação ambiental crescente, há a necessidade cada vez maior de eficácia e racionalidade para a utilização de adubos nitrogenados visando maior produtividade na agricultura (FRANCO; SARAIVA NETO, 2007).

Considerando ainda que o Brasil detém o maior rebanho comercial bovino do mundo, com um registro de abate em 2011 superior a 21 milhões de cabeças além de projeções de aumento de produção para os próximos anos (BRASIL, 2012), o que gera, conseqüentemente, uma alta produção de resíduos proveniente do abate e do processamento da carne, há a preocupação em sugerir a melhor forma de utilização destes resíduos, de forma que esses não venham a comprometer o equilíbrio natural do ambiente, bem como promover uma eficiente ciclagem dos nutrientes neles contidos. Um dos resíduos da indústria frigorífica é o conteúdo do rumem bovino. Segundo Mourales et al. (2006) cada animal bovino gera cerca de $25 \mathrm{~kg}$ no momento do abate.

Uma alternativa para a destinação final desse resíduo seria a sua utilização como fonte de adubação orgânica na agricultura o qual além de ser uma fonte de nutrientes às plantas e atuar como condicionador do solo têm se constituído como uma alternativa viável na preservação e qualidade ambiental (FREITAS et al., 2010).

A utilização de resíduos orgânicos tem ganhado destaque no cenário internacional, no entanto enfatiza-se avaliar os efeitos da sua utilização, devido a possibilidade desses resíduos conterem substâncias ou elementos orgânicos ou inorgânicos potencialmente tóxicos, principalmente quando se refere a lixiviação de N (LISBOA, 2007; PIRES; MATTIAZZO, 2008).

$\mathrm{O} \mathrm{N}$ apresenta um potencial poluidor, pois o nitrato $\left(\mathrm{NO}_{3}^{-}\right)$e o amônio $\left(\mathrm{NH}_{4}^{+}\right)$, em níveis excessivos são prejudiciais à saúde humana $\mathrm{e}$ animal. Sendo assim, o N no solo oriundo de adubação orgânica tem causado preocupação, devido ao potencial que este elemento apresenta de contaminação de águas subterrâneas pela lixiviação do $\mathrm{NO}_{3}^{-}$, tornando assim, de extrema importância o conhecimento das perdas de $\mathrm{N}$ do solo através desse 
processo (COELHO, et al., 2000; OLIVEIRA et al., 2001; SANTOS; MENEZES; BENITTES, 2009). Além da possibilidade de contaminação das águas, a lixiviação também coloca o $\mathrm{N}$ fora do alcance das raízes das plantas, diminuindo a eficiência da adubação nitrogenada, seja ela orgânica ou mineral.

O objetivo desse trabalho foi avaliar a disponibilidade e lixiviação de $\mathrm{N}$ no solo após a aplicação de diferentes doses de resíduo ruminal bovino (RRB), além de comparar com uma adubação nitrogenada mineral feita com uréia e determinar o risco potencial de contaminação de águas subterrâneas por $\mathrm{N}\left(\mathrm{NO}_{3}^{-} \mathrm{e} \mathrm{NH}_{4}^{+}\right)$em dois solos típicos do município de Pontes e Lacerda-MT.

\section{Material e Métodos}

O experimento foi conduzido na UNEMAT Universidade do Estado de Mato Grosso, Campus de Pontes e Lacerda, em casa de vegetação de dezembro de 2009 a março de 2010.
Os solos utilizados neste estudo foram classificados conforme o Sistema Brasileiro de Classificação de Solos (EMBRAPA, 2006) como Argissolo Vermelho-Amarelo eutrófico (PVAe) e Latossolo Vermelho distrófico (LVd), coletados nas coordenadas S $15^{\circ} 19^{\prime}$; W $059^{\circ} 14^{\prime}$ e S $15^{\circ} 20^{\prime}$; $\mathrm{W} 059^{\circ} 14^{\prime}$, respectivamente. Alguns atributos químicos e físicos dos solos relacionado à fertilidade, tais como: $\mathrm{pH}$ em água; $\mathrm{pH}$ em $\mathrm{KCl} ; \mathrm{Ca}^{2+} ; \mathrm{Mg}^{2+} \mathrm{e}$ $\mathrm{Al}^{3+}$ (extração com $\mathrm{KCl} 1 \mathrm{~mol} \mathrm{~L}-1$ ); acidez potencial (solução SMP); P e K ${ }^{+}$(extração com Mehlich 1); matéria orgânica (oxidação via úmida com $\mathrm{K}_{2} \mathrm{Cr}_{2} \mathrm{O}_{7}$ $0,4 \mathrm{~mol} \mathrm{~L}^{-1}$ ); textura (método da pipeta); densidade (método do anel volumétrico) e volume total de poros (VTP) foram determinadas conforme Embrapa (1997), o Delta $\mathrm{pH}(\Delta \mathrm{pH})$ foi calculado pela seguinte equação: $\mathrm{pH}_{\mathrm{KCl}}-\mathrm{pH}_{\mathrm{H} 2 \mathrm{O}}$ de acordo com Mekaru e Uehara (1972). As caracterizações químicas (Tabela 1) e físicas (Tabela 2) foram realizadas a cada $10 \mathrm{~cm}$ até a profundidade de $40 \mathrm{~cm}$.

Tabela 1. Atributos químicos do Argissolo Vermelho-Amarelo eutrófico (PVAe) e do Latossolo Vermelho distrófico (LVd) do município de Pontes e Lacerda - MT utilizados neste estudo.

\begin{tabular}{|c|c|c|c|c|c|c|c|c|c|}
\hline \multirow{3}{*}{ Atributos } & \multirow{3}{*}{ Unidade } & \multicolumn{8}{|c|}{ Profundidade $(\mathrm{cm})$} \\
\hline & & \multicolumn{4}{|c|}{ PVAe } & \multicolumn{4}{|c|}{ LVd } \\
\hline & & $0-10$ & $10-20$ & $20-30$ & $30-40$ & $0-10$ & $10-20$ & $20-30$ & $30-40$ \\
\hline $\mathrm{pH} \mathrm{H}_{2} \mathrm{O}$ & - & 6,3 & 6,7 & 6,8 & 7,0 & 6,4 & 6,5 & 6,2 & 5,6 \\
\hline $\mathrm{pH} \mathrm{KCl}$ & - & 5,6 & 5,7 & 5,8 & 5,7 & 5,7 & 5,6 & 5,0 & 4,3 \\
\hline$\Delta \mathrm{pH}$ & - & $-0,7$ & $-1,0$ & $-1,0$ & $-1,3$ & $-0,7$ & $-0,9$ & $-1,2$ & $-1,3$ \\
\hline $\mathrm{MO}^{1}$ & $\mathrm{~g} \mathrm{~kg}^{-3}$ & 13,93 & 7,47 & 3,83 & 1,61 & 14,1 & 10,7 & 8,1 & 5,0 \\
\hline $\mathrm{P}$ & & 6,7 & 3,9 & 7,2 & 4,6 & 2,3 & 1,1 & 1,0 & 1,8 \\
\hline $\mathrm{K}^{+}$ & $\mathrm{mg} \mathrm{dm}^{-3}$ & 30,2 & 16,6 & 16,6 & 18,1 & 102,4 & 87,3 & 91,9 & 100,9 \\
\hline $\mathrm{Ca}^{2+}$ & & 4,1 & 3,6 & 2,3 & 1,8 & 2,3 & 2,4 & 1,5 & 0,7 \\
\hline $\mathrm{Mg}^{2+}$ & छ & 0,5 & 0,1 & 0,2 & 0,4 & 1,4 & 1,1 & 0,5 & 1,0 \\
\hline $\mathrm{Al}^{3+}$ & $0^{\circ}$ & 0,0 & 0,0 & 0,0 & 0,0 & 0,0 & 0,0 & 0,0 & 0,3 \\
\hline $\mathrm{H}+\mathrm{Al}$ & हี & 1,7 & 1,1 & 1,1 & 0,9 & 1,7 & 1,6 & 1,9 & 2,6 \\
\hline $\mathrm{SB}^{2}$ & & 4,6 & 3,7 & 2,5 & 2,2 & 4,0 & 3,7 & 2,3 & 1,9 \\
\hline $\mathrm{CTC}^{3}$ efetiva & & 4,6 & 3,7 & 2,5 & 2,2 & 4,0 & 3,7 & 2,3 & 2,2 \\
\hline CTC total & & 6,4 & 4,8 & 3,6 & 3,1 & 5,7 & 5,3 & 4,1 & 4,5 \\
\hline $\mathrm{V}^{4}$ & $\%$ & 73,0 & 76,4 & 70,3 & 70,9 & 69,7 & 69,5 & 54,8 & 42,4 \\
\hline
\end{tabular}

${ }^{1} \mathrm{MO}$ - Matéria Orgânica; ${ }^{2} \mathrm{SB}$ - Soma de bases; ${ }^{3} \mathrm{CTC}$ - Capacidade de troca de cátions; ${ }^{4} \mathrm{~V}$ - Saturação por bases.

Fonte: Elaboração dos autores. 
Tabela 2. Características físicas do Argissolo Vermelho-Amarelo eutrófico (PVAe) e do Latossolo Vermelho distrófico (LVd) do município de Pontes e Lacerda - MT utilizados neste estudo.

\begin{tabular}{|c|c|c|c|c|c|c|}
\hline \multirow{2}{*}{ Solo } & Prof. & Areia & Silte & Argila & Densidade do solo & VTP \\
\hline & $\mathrm{cm}$ & & $\mathrm{g} \mathrm{kg}^{-1}$ & & $\mathrm{~g} \mathrm{~cm}^{-3}$ & $\%$ \\
\hline \multirow{4}{*}{$\sum_{a}^{0}$} & $0-10$ & 627,4 & 249,4 & 123,3 & 1,38 & 41,8 \\
\hline & $10-20$ & 751,3 & 67,7 & 180,5 & 1,63 & 31,2 \\
\hline & $20-30$ & 719,8 & 108,4 & 171,8 & 1,58 & 41,3 \\
\hline & $30-40$ & 682,5 & 65,6 & 251,9 & 1,59 & 40,9 \\
\hline \multirow{5}{*}{$\bar{J}$} & Média & 695,2 & 122,7 & 181,8 & 1,54 & 38,8 \\
\hline & $0-10$ & 751,8 & 67,7 & 180,5 & 1,45 & 42,2 \\
\hline & $10-20$ & 751,8 & 67,7 & 180,5 & 1,54 & 38,7 \\
\hline & $20-30$ & 598,6 & 99,8 & 301,6 & 1,44 & 37,7 \\
\hline & $30-40$ & 650,6 & 72,0 & 277,4 & 1,40 & 39,4 \\
\hline & Média & 688,2 & 76,8 & 235,0 & 1,46 & 39,5 \\
\hline
\end{tabular}

Fonte: Elaboração dos autores.

O RRB utilizado nesse experimento foi coletado na indústria frigorífica do município de Pontes e Lacerda - MT. A análise de N total do RRB seguiu a metodologia proposta por Bremner (1996), as demais análises relacionadas ao valor agronômico do resíduo tais como $\mathrm{pH}$ em água, condutividade elétrica, teor de sódio, teor de carbono (matéria orgânica), umidade atual do resíduo, capacidade de retenção de água do resíduo, teor de $\mathrm{NH}_{4}^{+}$, teor de $\mathrm{NO}_{3}{ }^{-}$, teores de macronutrientes $\left(\mathrm{P}, \mathrm{K}^{+}, \mathrm{Na}^{+}, \mathrm{Ca}^{2+}\right.$, $\mathrm{Mg}^{2+}$ e S), teores de micronutrientes $(\mathrm{Cu}, \mathrm{Fe}, \mathrm{Zn}$, $\mathrm{Mn}$ e B), foram realizadas em função do emprego da digestão nítrico-perclórica conforme metodologia descrita por Tedesco et al. (1995) e os resultados podem ser observados na Tabela 3 .
Para avaliar a lixiviação de $\mathrm{NO}_{3}^{-}$e $\mathrm{NH}_{4}^{+}$ foram preparadas colunas de percolação constituídas de tubos de PVC de $40 \mathrm{~cm}$, sendo essas colunas preenchidas com PVAe e LVd, os quais receberam doses de RRB incorporados a 10 $\mathrm{cm}$ de profundidade. As doses foram calculados para fornecer o equivalente a $0 ; 50 ; 100$ e $400 \mathrm{~kg}$ de $\mathrm{N} \mathrm{ha}^{-1}$, sendo os tratamentos denominados, respectivamente, de RRB0; RRB50; RRB100 e RRB400. Adicionalmente foi realizado um tratamento com uréia para fornecer o equivalente a $400 \mathrm{~kg}$ de $\mathrm{N} \mathrm{ha}^{-1}$ (U400). Todos os tratamentos foram realizados com 4 repetições, totalizando 40 colunas de solo.

Tabela 3. Características do resíduo ruminal bovino (RRB) coletado em frigorífico do município de Pontes e Lacerda - MT.

\begin{tabular}{lcclcc}
\hline Atributos & Unidade & Resultado & Atributos & Unidade & Resultado \\
\hline $\mathrm{pH} \mathrm{H} \mathrm{H}_{2} \mathrm{O}$ & - & 6,2 & Potássio $\left(\mathrm{K}^{+}\right)$Total & $\mathrm{g} \mathrm{kg}^{-1}$ & 1,0 \\
Cond. elétrica & $\mathrm{dS} \mathrm{m}^{-1}$ & 1,9 & Sódio $\left(\mathrm{Na}^{+}\right)$ & $\mathrm{g} \mathrm{kg}^{-1}$ & 1,8 \\
Capacidade de retenção de água & $\mathrm{mL} \mathrm{g}^{-1}$ & 4,2 & Cálcio $\left(\mathrm{Ca}^{2+}\right)$ & $\mathrm{g} \mathrm{kg}^{-1}$ & 5,5 \\
Umidade Atual & $\mathrm{g} \mathrm{kg}^{-1}$ & 928,4 & Magnésio $\left(\mathrm{Mg}^{2+}\right)$ & $\mathrm{g} \mathrm{kg}^{-1}$ & 0,5 \\
Carbono Total & $\mathrm{g} \mathrm{kg}^{-1}$ & 460 & Enxofre $(\mathrm{S})$ & $\mathrm{g} \mathrm{kg}^{-1}$ & 1,4 \\
Matéria Orgânica & $\mathrm{g} \mathrm{kg}^{-1}$ & 910 & Boro $(\mathrm{B})$ & $\mathrm{mg} \mathrm{kg}^{-1}$ & 5,8 \\
Nitrogênio (N) Total & $\mathrm{g} \mathrm{kg}^{-1}$ & 14,3 & Cobre $(\mathrm{Cu})$ & $\mathrm{mg} \mathrm{kg}^{-1}$ & 122 \\
$\mathrm{~N}$ - amônio & $\mathrm{mg} \mathrm{kg}^{-1}$ & 60 & Ferro $(\mathrm{Fe})$ & $\mathrm{g} \mathrm{kg}^{-1}$ & 1,42 \\
$\mathrm{~N}$ - nitrato & $\mathrm{mg} \mathrm{kg}^{-1}$ & 14 & Manganês $(\mathrm{Mn})$ & $\mathrm{mg} \mathrm{kg}^{-1}$ & 90 \\
Fósforo (P) Total & $\mathrm{g} \mathrm{kg}^{-1}$ & 3,8 & Zinco $(\mathrm{Zn})$ & $\mathrm{mg} \mathrm{kg}^{-1}$ & 91 \\
\hline
\end{tabular}

Fonte: Elaboração dos autores. 
A quantidade necessária de RRB para atender as doses dos tratamentos: RRB0, RRB50, RRB100 e RRB400 foram calculadas com base no teor de $\mathrm{N}$ total do RRB considerando-se que apenas 33\% seriam disponibilizados no primeiro ano (EICHLER et al., 2008), sendo equivalente à $0 ; 10 ; 21 ; 84 \mathrm{Mg}$ ha $^{-1}$ de RRB, respectivamente. O tratamento com uréia foi calculado considerando-se um teor de $46 \%$ de $\mathrm{N}$ nesse fertilizante correspondendo à aplicação de $0,86 \mathrm{Mg} \mathrm{ha}^{-1}$.
As colunas de solo foram preparadas com tubos de PVC de $10 \mathrm{~cm}$ de diâmetro e $45 \mathrm{~cm}$ de altura (Figura 1a), coletadas diretamente no solo, tomando-se o cuidado para manter a estrutura do solo indeformada. A irrigação das colunas durante o período do experimento foi realizada por gotejamento controlada por equipos de infusão gravitacional (Figura 1b). No fundo da coluna foi conectada uma mangueira, a qual foi ligada a um frasco para a coleta do lixiviado (Figura 1c), seguindo metodologia semelhante à utilizada por Cembranelli (2006).

Figura 1. Colunas de solos (a); equipos para dosagem do gotejamento (b); frascos para coleta de lixiviado (c).
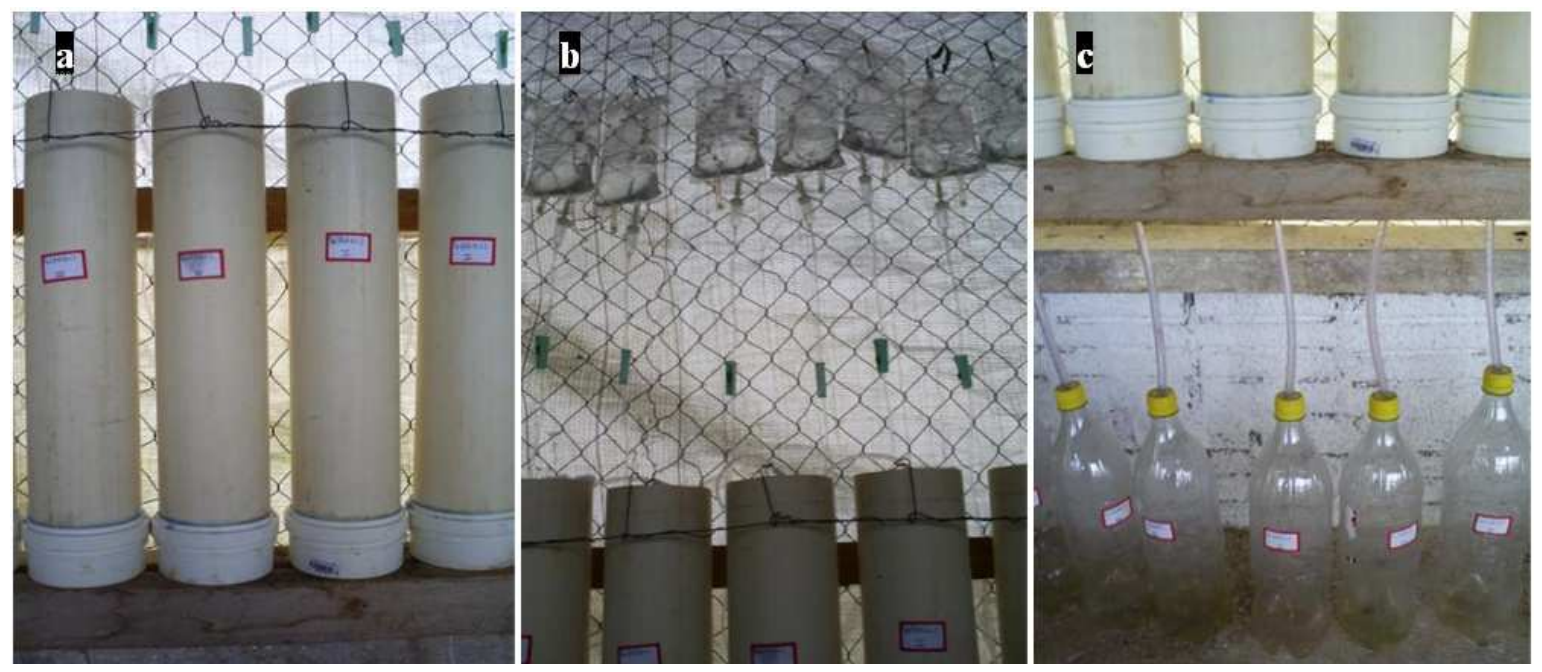

Fonte: Elaboração dos autores.

A irrigação das colunas foi realizada semanalmente mediante a adição de $385 \mathrm{~mL}$ de água destilada simulando a quantidade de chuva durante o período de precipitação mais intensa no município referente aos meses de novembro à março. Considerou-se a precipitação média do município de Pontes e Lacerda igual a $1.500 \mathrm{~mm}$ ano $^{-1}$ (FERREIRA, 2001).

Todas as colunas foram submetidas a uma irrigação inicial de água até completar o volume total de poros, equivalente a 1,223 e 1,242 mL, respectivamente no PVAe e no $\mathrm{LVd}$, com o objetivo de homogeneizar o teor de umidade do solo de todas as colunas. Após a adição de RRB iniciou-se a irrigação das colunas de solo, tomando-se o cuidado para manter o gotejamento em torno de 0,5 gotas por segundo e sempre no centro da coluna no intuito de se evitar um fluxo preferencial. Toda semana era anotado o volume de lixiviado e coletada uma alíquota de $5 \mathrm{~mL}$ de cada amostra sendo conservada em frasco de vidro âmbar de $100 \mathrm{~mL}$ à $4^{\circ} \mathrm{C}$. Em cada frasco de vidro foi adicionado uma gota de ácido clorídrico para melhor conservação da amostra até o procedimento de determinação do $\mathrm{NO}_{3}{ }^{-} \mathrm{e} \mathrm{NH}_{4}^{+}$ (SANTA CATARINA, 2008).

Após o período experimental coletou-se amostras de solo das colunas a cada $10 \mathrm{~cm}$ de profundidade, formando um total de 160 amostras de solo, as quais 
foram encaminhadas ao laboratório para análises. A coleta do lixiviado procedida semanalmente em cada tratamento formou 40 amostras. As determinações dos teores de $\mathrm{NO}_{3}^{-}$e $\mathrm{NH}_{4}^{+}$no solo e no lixiviado foram realizadas seguindo metodologia baseada em Bremner e Keeney (1966) e os resultados foram submetidos à análise de variância utilizando-se o programa estatístico Sisvar sendo as médias comparadas pelo teste de Scott e Knott (1974) (p < $0,05)$. Além disso, procedeu-se, quando pertinentes, as análises de regressão dos teores de $\mathrm{NO}_{3}{ }^{-}$e $\mathrm{NH}_{4}{ }^{+}$ em função das doses de RRB a fim de se obter um modelo matemático de predição dessas variáveis em função das doses aplicadas.

\section{Resultados e Discussão}

Teores de $\mathrm{NO}_{3}^{-}$e $\mathrm{NH}_{4}^{+}$no solo

As maiores doses de resíduo aplicado (RRB100 e RRB400) resultaram em teores mais altos de $\mathrm{NO}_{3}^{-}$no $\mathrm{LVd}$, sendo também superior em relação ao $\mathrm{NH}_{4}^{+}$(Figura 2). Esse resultado indica que houve mineralização e posterior nitrificação, incrementando os teores de $\mathrm{NO}_{3}^{-}$ao solo com efeito significativo da dose aplicada. Boeira, Ligo e Dynia (2002) estudando o efeito do lodo de esgoto encontraram resultados semelhantes demonstrando efeito significativo entre o acúmulo de $\mathrm{N}$ mineral e a quantidade de $\mathrm{N}$ orgânico aplicado ao solo, com predominância de $\mathrm{NO}_{3}^{-}$aos 105 dias de incubação.

Figura 2. Teor de $\mathrm{NO}_{3}^{-}$e $\mathrm{NH}_{4}^{+}\left(\mathrm{mg} \mathrm{kg}^{-1}\right)$ no Latossolo Vermelho distrófico (LVd) e no Argissolo Vermelho Amarelo eutrófico do município de Pontes e Lacerda - MT, em função dos tratamentos. RRB = Resíduo ruminal bovino $\left(0 ; 50 ; 100\right.$ e $400 \mathrm{~kg}$ de $\left.\mathrm{N} \mathrm{ha}^{-1}\right) ; \mathrm{U}=$ Uréia $\left(400 \mathrm{~kg}\right.$ de $\left.\mathrm{N} \mathrm{ha}^{-1}\right)$. Letras minúsculas comparam os tratamentos em cada variável, letras maiúsculas comparam as variáveis dentro de cada tratamento (teste de Scott-Knott, $\mathrm{p}<0,05$ ).
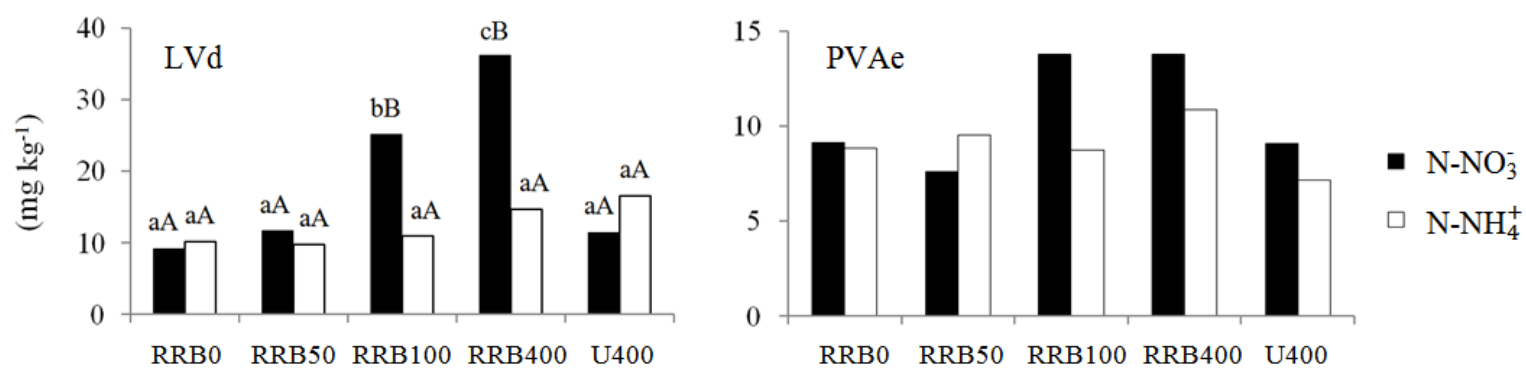

Fonte: Elaboração dos autores.

Na Figura 3 observa-se a regressão estabelecida entre a dose de aplicação do resíduo e a disponibilidade de $\mathrm{NO}_{3}^{-}$no $\mathrm{LVd}$ na profundidade de 0-40 cm. Por derivação da regressão observouse que o máximo de disponibilização de $\mathrm{NO}_{3}^{-}$pelo fornecimento de RRB seria em torno da dose de 72 $\mathrm{Mg} \mathrm{ha}^{-1}$, resultando em um teor de $38,47 \mathrm{mg} \mathrm{kg}^{-1}$ de $\mathrm{NO}_{3}{ }^{-}$no solo, ou seja, algo em torno de 224,6 $\mathrm{kg} \mathrm{ha}^{-1}$ considerando a densidade média do solo, na camada de 0-40 $\mathrm{cm}$ igual a $1,46 \mathrm{~g} \mathrm{~cm}^{-3}$ (Tabela 2).
Uma aplicação exagerada do resíduo nesse solo a partir da dose de $72 \mathrm{Mg} \mathrm{ha}^{-1}$ provocaria então uma redução na disponibilidade de $\mathrm{NO}_{3}{ }^{-}$no solo dentro do período estudado, o que pode ser atribuído à um possível estresse causado as comunidades microbianas. Como mencionado por Martines et al. (2006) tais fatores são as flutuações na temperatura, os extremos de potencial hídrico, extremos de $\mathrm{pH}$, distúrbios físicos do solo, mudanças nas trocas gasosas, decréscimo no suprimento de nutrientes e a presença de predadores e antagonistas. 
Figura 3. Regressão entre a disponibilidade de $\mathrm{NO}_{3}{ }^{-}$no solo e a dose de aplicação de Resíduo Ruminal Bovino (RBB) no Latossolo Vermelho distrófico (LVd) do município de Pontes e Lacerda - MT.

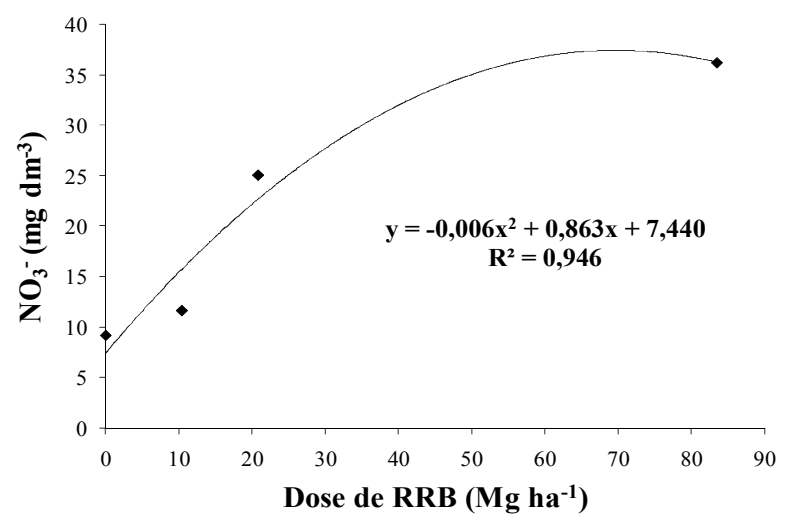

Fonte: Elaboração dos autores.

Não houve diferença significativa dos teores de $\mathrm{NO}_{3}{ }^{-}$entre a testemunha (RRB0) e o RRB50 no LVd (Figura 2), podendo ser atribuída à menor dose de $\mathrm{N}$ aplicada no tratamento RRB50 (50 kg de $\mathrm{N} \mathrm{ha}^{-1}$ ). Esta dose não teria sido suficiente para incrementar os teores de $\mathrm{NO}_{3}^{-}$no solo dentro do período estudado, em quantidade superior à capacidade natural que este solo apresenta de disponibilização do $\mathrm{N}$ decorrente de seus teores de MO (Tabela 1). Lisboa et al. (2007) não encontraram diferenças nos teores de $\mathrm{NO}_{3}^{-}$em um LVd pela adição de doses de $120 \mathrm{~kg}$ de $\mathrm{N} \mathrm{ha}^{-1}$ em colunas de solo tratados com adubos orgânicos e sintéticos em relação à testemunha em um período de 90 dias. Esse fato evidencia a importância da MO presente no solo para a disponibilização de $\mathrm{N}$, contribuindo para a sustentação de sistemas produtivos de baixo nível tecnológico, conforme relatado por Cantarutti et al. (1999) em relação ao cultivo de pastagens.

O tratamento com uréia (U400) apesar de ter sido calculada para fornecer $400 \mathrm{~kg}$ de $\mathrm{N} \mathrm{ha}^{-1}$, não influenciou nos teores de $\mathrm{NO}_{3}{ }^{-}$e $\mathrm{NH}_{4}^{+}$no $\mathrm{LVd}$ em relação à testemunha (Figura 2). Esse fato pode ser associado ao alto potencial de volatilização da amônia $\left(\mathrm{N}-\mathrm{NH}_{3}\right)$ em condições de altas temperaturas e umidade do solo pela aplicação da uréia (MARTHA JÚNIOR et al., 2007), visto que o período do experimento ocorreu durante o verão, caracterizado por temperaturas altas associado ainda a alta condição de umidade as quais as colunas de solo foram submetidas. Cabezas e Souza (2008) constataram processo semelhante com perdas de $76,8 \%$ de $\mathrm{N}$ aplicado na forma de uréia pela aplicação de doses de $54,3 \mathrm{~kg}$ de $\mathrm{N} \mathrm{ha}^{-1} \mathrm{em}$ Latossolo Vermelho ácrico.

No PVAe não houve diferença entre os teores de $\mathrm{NO}_{3}^{-}$e $\mathrm{NH}_{4}^{+}$, não sendo observado, também, diferenças entre os tratamentos (Figura 2). Possivelmente não houve a mineralização esperada dos tratamentos que receberam as doses de RRB tal qual verificada no LVd. Esses resultados podem indicar que características dos solos podem exercer efeito sobre a mineralização do resíduo e disponibilização de $\mathrm{N}$, neste caso observa-se principalmente que o PVAe apresentou maior densidade (Tabela 2) o que possivelmente dificultou a percolação da água, retendo a umidade por mais tempo no solo, o que pode ter prejudicado a mineralização do RRB no PVAe, quando comparado ao LVd.

No tratamento que recebeu doses de Uréia (U400), no PVAe, ocorreu comportamento semelhante ao observado no LVd (Figura 2), não havendo diferença com a testemunha, sugerindo- 
se então, que tenha ocorrido perdas de $\mathrm{N}$ por volatilização da $\mathrm{N}-\mathrm{NH}_{3}$.

No perfil do $\mathrm{LVd}$, de modo geral não foram observadas diferenças nos teores de $\mathrm{NO}_{3}{ }^{-}$e $\mathrm{NH}_{4}^{+}$ em profundidade que demonstrasse lixiviação desse elemento em decorrência da aplicação do RRB (Tabela 4), sendo os mais altos teores observados apenas na camada de 0 a $10 \mathrm{~cm}$, que foi a profundidade em que o RRB foi incorporado. No entanto, os teores de $\mathrm{NO}_{3}{ }^{-}$foram mais elevados na profundidade de 30 a $40 \mathrm{~cm}$ no tratamento RRB100. É possível que, neste caso o fluxo de água na coluna de solo não ter sucedido de maneira adequada o que ocasionou acúmulo de $\mathrm{NO}_{3}^{-}$nesta profundidade, visto que nos outros tratamentos não ocorreram processo semelhante.

Tabela 4. Teores de $\mathrm{NO}_{3}^{-}$e $\mathrm{NH}_{4}^{+}\left(\mathrm{mg} \mathrm{kg}^{-1}\right)$ no perfil do Latossolo Vermelho distrófico (LVd) do município de Pontes e Lacerda - MT, em função dos tratamentos ${ }^{1}$.

\begin{tabular}{|c|c|c|c|c|c|c|}
\hline \multirow{2}{*}{ Variável } & \multirow{2}{*}{$\begin{array}{c}\text { Prof. } \\
\mathrm{cm}\end{array}$} & \multicolumn{5}{|c|}{ TRATAMENTOS } \\
\hline & & RRB0 & RRB50 & RRB100 & RRB400 & U400 \\
\hline \multirow{4}{*}{$\dot{o}^{m}$} & $0-10$ & $12,18 \mathrm{a}$ & $21,04 a$ & $32,34 b$ & $67,15 b$ & $13,69 a$ \\
\hline & $10-20$ & $10,27 \mathrm{a}$ & $10,28 \mathrm{a}$ & $23,04 \mathrm{a}$ & $29,12 \mathrm{a}$ & $13,71 \mathrm{a}$ \\
\hline & $20-30$ & $7,40 \mathrm{a}$ & $7,81 \mathrm{a}$ & $14,65 \mathrm{a}$ & $29,30 \mathrm{a}$ & $7,82 \mathrm{a}$ \\
\hline & $30-40$ & $7,04 \mathrm{a}$ & $7,60 \mathrm{a}$ & $30,27 \mathrm{~b}$ & $19,22 \mathrm{a}$ & $10,62 \mathrm{a}$ \\
\hline \multirow{4}{*}{$\stackrel{+}{\mathbf{Z}}^{+}$} & $0-10$ & $8,95 \mathrm{a}$ & $9,51 \mathrm{a}$ & $13,13 a$ & $31,38 b$ & $16,16 \mathrm{a}$ \\
\hline & $10-20$ & $10,08 \mathrm{a}$ & $9,34 \mathrm{a}$ & $9,49 a$ & $9,88 \mathrm{a}$ & $12,55 \mathrm{a}$ \\
\hline & $20-30$ & $12,37 \mathrm{a}$ & $10,07 \mathrm{a}$ & $12,56 \mathrm{a}$ & $7,78 \mathrm{a}$ & $17,12 \mathrm{a}$ \\
\hline & $30-40$ & $9,32 \mathrm{a}$ & $10,09 \mathrm{a}$ & $8,37 \mathrm{a}$ & $9,89 \mathrm{a}$ & $20,18 \mathrm{a}$ \\
\hline
\end{tabular}

Médias seguidas de mesma letra na coluna não diferem estatisticamente pelo teste de Scott-Knott $(\mathrm{p}<0,05)$. ${ }^{1} \mathrm{RRB}=\mathrm{Resíduo}$ ruminal bovino (0;50;100 e $400 \mathrm{~kg}$ de $\mathrm{N}$ ha-1); U = Uréia (400 kg de $\mathrm{N}$ ha-1).

Fonte: Elaboração dos autores.

Por outro lado, os teores de $\mathrm{NO}_{3}{ }^{-}$em profundidade no PVAe evidenciaram a ocorrência de lixiviação (Tabela 5). O tratamento RRB100 apresentou maior teor de $\mathrm{NO}_{3}^{-}$na profundidade de 10 a $20 \mathrm{~cm} \mathrm{e} \mathrm{o}$ tratamento RRB400 na profundidade de 30 a 40 $\mathrm{cm}$ devido à maior carga de resíduo aplicada nesses tratamentos que possivelmente contribuíram com aumento na disponibilidade de $\mathrm{N}$, visto que nos tratamentos RRB0 e RRB50 não houve diferenças nos teores de $\mathrm{NO}_{3}{ }_{3}^{-}$no perfil do solo (Tabela 5), apesar de não terem sido observadas diferenças estatísticas entre os tratamentos (Figura 2). Aguiar et al. (2006) relataram lixiviação de $\mathrm{NO}_{3}^{-}$em um Argissolo Acinzentado devido a característica negativa da superfície dos colóides do solo, a qual repele essa molécula, fazendo-a permanecer livre na solução do solo, facilitando sua lixiviação para camadas mais profundas. No presente trabalho, tanto o LVd quanto o PVAe apresentaram característica negativa da superfície dos colóides do solo $(\Delta \mathrm{pH})$, o que favorece nos dois solos a lixiviação do $\mathrm{NO}_{3}^{-}$. 
Tabela 5. Teores de $\mathrm{NO}_{3}^{-}$e $\mathrm{NH}_{4}^{+}\left(\mathrm{mg} \mathrm{kg}^{-1}\right)$ no perfil do Argissolo Vermelho-Amarelo eutrófico (PVAe) do município de Pontes e Lacerda - MT, em função dos tratamentos ${ }^{1}$.

\begin{tabular}{|c|c|c|c|c|c|c|}
\hline \multirow{2}{*}{ Variável } & \multirow{2}{*}{$\begin{array}{c}\text { Prof. } \\
\mathrm{cm}\end{array}$} & \multicolumn{5}{|c|}{ TRATAMENTOS } \\
\hline & & RRB0 & RRB50 & RRB100 & RRB400 & U400 \\
\hline \multirow{4}{*}{$\dot{0}^{m}$} & $0-10$ & $9,03 a$ & $8,83 a$ & $14,15 \mathrm{a}$ & $13,55 \mathrm{a}$ & $5,68 \mathrm{a}$ \\
\hline & $10-20$ & $5,78 \mathrm{a}$ & $5,40 \mathrm{a}$ & $20,05 b$ & $10,33 a$ & $8,63 \mathrm{a}$ \\
\hline & $20-30$ & $11,88 \mathrm{a}$ & $8,25 \mathrm{a}$ & $12,48 \mathrm{a}$ & $8,43 a$ & $11,53 \mathrm{a}$ \\
\hline & $30-40$ & $9,80 \mathrm{a}$ & $7,85 \mathrm{a}$ & $8,43 \mathrm{a}$ & $22,70 \mathrm{~b}$ & $10,55 \mathrm{a}$ \\
\hline \multirow{4}{*}{$\stackrel{\vec{z}}{+}^{+}$} & $0-10$ & $12,05 a$ & $12,98 \mathrm{a}$ & $9,75 a$ & $17,53 b$ & $3,83 a$ \\
\hline & $10-20$ & $7,45 \mathrm{a}$ & $11,33 a$ & $8,20 \mathrm{a}$ & $9,75 \mathrm{a}$ & $5,55 \mathrm{a}$ \\
\hline & $20-30$ & $11,05 \mathrm{a}$ & $6,68 \mathrm{a}$ & $10,70 \mathrm{a}$ & $9,18 \mathrm{a}$ & $8,03 a$ \\
\hline & $30-40$ & $4,78 \mathrm{a}$ & $7,10 \mathrm{a}$ & $6,33 \mathrm{a}$ & $6,90 \mathrm{a}$ & $11,10 \mathrm{a}$ \\
\hline
\end{tabular}

Médias seguidas de mesma letra na coluna não diferem estatisticamente pelo teste de $\operatorname{Scott-Knott~}(p<0,05)$. ${ }^{1} \mathrm{RRB}=$ Resíduo ruminal bovino (0;50;100 e $400 \mathrm{~kg}$ de $\mathrm{N}$ ha-1); $\mathrm{U}=$ Uréia (400 kg de $\mathrm{N}$ ha-1).

Fonte: Elaboração dos autores.

$\mathrm{O} \mathrm{NH}_{4}^{+}$não apresentou mobilidade no perfil do PVAe, sendo os teores iguais em todas as profundidades amostradas, exceto na profundidade de $0-10 \mathrm{~cm}$ no tratamento RRB400 no qual este foi mais elevado (Tabela 5). Estes resultados sugerem que o PVAe apresenta capacidade de retenção de $\mathrm{NH}_{4}^{+}$, enquanto o $\mathrm{NO}_{3}{ }^{-}$seja mais facilmente lixiviado, evidenciando o comportamento diferenciado destes elementos no perfil do solo.

O tratamento U400 não influenciou significativamente os teores de $\mathrm{NO}_{3}^{-}$ou $\mathrm{NH}_{4}^{+}$no perfil do solo mesmo sendo possível observar um gradual aumento nas camadas subsuperficiais (Tabela 5), no entanto destaca-se a possibilidade de ocorrência de grandes perdas de $\mathrm{N}$ por volatilização em função da forma de aplicação da uréia (CANTARELLA, 2007), haja vista que este tratamento não apresentou efeito na disponibilidade de $\mathrm{N}$ no solo quando comparado aos tratamento testemunha (RRB0) e RRB50 (Figura 2). Dynia, Boeira e Souza (2006) constataram menores perdas de $\mathrm{NO}_{3}^{-}$em um Latossolo Vermelho distroférrico através de adubação mineral nitrogenada parcelada em relação à testemunha e ao tratamento com lodo de esgoto, neste caso, o experimento foi realizado sob sucessão de 3 anos de cultivos de milho, no qual foi observado maior desenvolvimento radicular no tratamento com adubação mineral e, conseqüentemente, maior absorção do N. Primavesi et al. (2006) determinaram que doses parceladas de até cinco vezes de uréia para a dose de $500 \mathrm{~kg} \mathrm{ha}^{-1}$, no período das chuvas em pastagem de capim coastcross não proporcionaram perdas significativas de $\mathrm{NO}_{3}{ }^{-}$para o lençol freático.

Em se tratando das diferenças entre adubação orgânica e mineral, com relação ao comportamento do $\mathrm{N}$ no solo, deve-se admitir que a adubação mineral permite controlar com maior exatidão a quantidade fornecida para as culturas. Fernandes e Libardi (2009) constataram que a lixiviação de $\mathrm{NO}_{3}^{-}$ proveniente de fertilizante mineral foi praticamente nula no primeiro cultivo de milho e, em geral, baixa durante o cultivo de braquiária e o segundo cultivo de milho. Sendo assim, as quantidades de $\mathrm{N}$ perdidas podem ser minimizadas, considerando que determinada cultura absorva os nutrientes fornecidos se atendidas adequadamente as suas exigências, pois no caso de excesso de nutrientes através da adubação, esses poderão ser perdidos mais facilmente.

\section{Teores de $\mathrm{NO}_{3}^{-}$e $\mathrm{NH}_{4}^{+}$no lixiviado}

No lixiviado do LVd não houve acúmulo de $\mathrm{NO}_{3}^{-}$por efeito de tratamento, não sendo observada nenhuma diferença entre os mesmos (Figura 4). 
Resultado diferente foi determinado por Boeira (2009) que observou progressivo aumento de $\mathrm{NO}_{3}^{-}+\mathrm{NO}_{2}^{-}$acumulado no lixiviado produzido pela incubação de lodo de esgoto de acordo com o aumento das doses aplicadas $(0,100,400$, e $800 \mathrm{~kg}$ de $\left.\mathrm{N} \mathrm{ha}^{-1}\right)$.

Figura 4. Teor de $\mathrm{NO}_{3}^{-}$e $\mathrm{NH}_{4}^{+}\left(\mathrm{mg} \mathrm{L}^{-1}\right)$ no lixiviado do Latossolo Vermelho distrófico (LVd) e do Argissolo Vermelho Amarelo eutrófico do município de Pontes e Lacerda - MT, em função dos tratamentos. RRB = Resíduo ruminal bovino $\left(0 ; 50 ; 100\right.$ e $400 \mathrm{~kg}$ de $\mathrm{N}$ ha $\left.^{-1}\right) ; \mathrm{U}=$ Uréia $\left(400 \mathrm{~kg} \mathrm{de} \mathrm{N} \mathrm{ha}^{-1}\right)$. Letras minúsculas comparam os tratamentos em cada variável, letras maiúsculas comparam as variáveis dentro de cada tratamento (teste de Scott-Knott, $\mathrm{p}<0,05$ ).
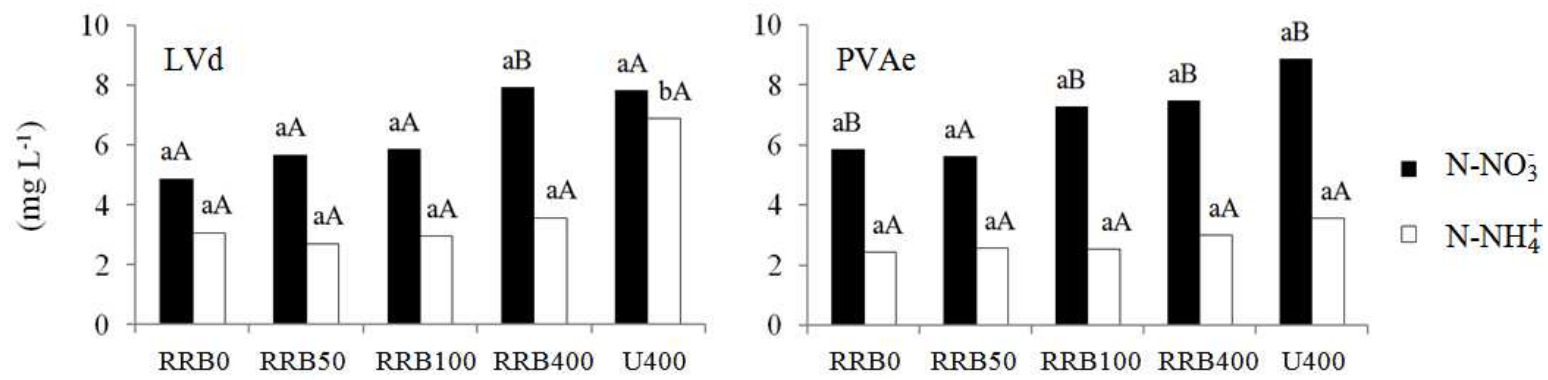

Fonte: Elaboração dos autores.

Quanto aos teores de $\mathrm{NH}_{4}{ }^{+}$no lixiviado do $\mathrm{LVd}$, também não houve diferença entre os tratamentos de RRB (Figura 4). Já o tratamento U400 apresentou teor de $\mathrm{NH}_{4}^{+}$superior aos demais (RRB0, RRB50, RRB100 e RRB400), podendo-se supor que logo após a aplicação da uréia nesse tratamento, tenha ocorrido perdas também por lixiviação. Esse processo indica que a aplicação de uréia além de apresentar perdas de $\mathrm{N}$ por volatilização é possível ocorrer significativas perdas também por lixiviação, o qual resultou em acúmulo de $\mathrm{NH}_{4}^{+}$no lixiviado coletado, tal como verificado por Araújo et al. (2004) que relataram que os tratamentos que receberam doses de uréia apresentaram uma alta lixiviação de $\mathrm{NH}_{4}^{+} \operatorname{logo}$ no início do experimento.

Diferença entre os teores de $\mathrm{NH}_{4}^{+} \mathrm{e} \mathrm{NO}_{3}^{-}$ no lixiviado no LVd foi observada apenas no tratamento RRB400 (Figura 4) no qual os teores de $\mathrm{NO}_{3}^{-}$foram superiores aos de $\mathrm{NH}_{4}^{+}$, com razoável tendência de maiores teores de $\mathrm{NO}_{3}{ }_{3}^{-}$nos demais tratamentos, apesar de não haver diferença estatística. Devido à maior disponibilidade de $\mathrm{NO}_{3}{ }^{-}$observada no tratamento RRB400 (Figura 2), justifica-se sua maior concentração no lixiviado em relação ao $\mathrm{NH}_{4}^{+}$, visto que há a predominância de cargas negativas nesse solo $(\Delta \mathrm{pH}<0)$ (Tabela 1$)$, o que favorece a lixiviação do $\mathrm{NO}_{3}^{-}$.

No lixiviado do PVAe foram evidenciados maiores teores de $\mathrm{NO}_{3}^{-}$em relação ao $\mathrm{NH}_{4}^{+}$(Figura 4), o qual se justifica devido a predominância de cargas líquidas negativas no solo $(\Delta \mathrm{pH}<0)$ (Tabela 1), ocorrendo então maior lixiviação de $\mathrm{NO}_{3}^{-}$mesmo não havendo diferenças entre esses elementos no solo (Figura 2), no entanto, diferente do observado no LVd que apresentou diferenças significativa apenas no tratamento em que houve maior disponibilidade de $\mathrm{NO}_{3}$.

Em relação ao $\mathrm{NH}_{4}^{+}$, Brito, Rolim e Pedrossa (2007) destacam o potencial do Argissolo em reter cátions no solo. No mesmo sentido, Andrade et al. (2005) apresentaram resultados no lixiviado dessa classe de solo em que os teores de $\mathrm{NO}_{3}^{-}$foram elevados em tratamento com fertilizante e efluente doméstico. Fey et al. (2010) concluíram que doses de dejeto suíno, pré-estabilizado em esterqueira por 120 dias, superiores a $60 \mathrm{~m}^{3} \mathrm{ha}^{-1}$ oferecem riscos de lixiviação de $\mathrm{NO}_{3}^{-}$principalmente no Argissolo, comparado ao Latossolo. Estes resultados 
encontrados na literatura são semelhantes aos observados neste estudo, em que os teores desses elementos no lixiviado apresentaram maior tendência de lixiviação de $\mathrm{NO}_{3}{ }^{-}$no PVAe, enquanto o $\mathrm{LVd}$ reteve melhor o $\mathrm{NO}_{3}^{-}$. Considerando que os dois solos estudados apresentaram atributos químicos semelhantes (Tabela 1), tais como CTC e o $\Delta \mathrm{pH}$, é possível inferir que uma maior lixiviação de $\mathrm{NO}_{3}^{-}$no PVAe esteja condicionada a atributos que não foram avaliados nesse estudo, assim sendo os óxidos de Ferro e Alumínio e materiais amorfos, além da mineralogia dos solos.

Oliveira, Vilela e Yarza (2000) estudando a adsorção de $\mathrm{NO}_{3}^{-}$em latossolo do cerrado, observaram que a adsorção do $\mathrm{NO}_{3}^{-}$também pode ocorrer quando a carga líquida é negativa, o que indica que as cargas positivas presentes são neutralizadas, pelo menos em parte, pelo nitrato, e não pelas cargas negativas adjacentes. Black e Waring (1979) verificaram que as propriedades do solo de diferentes mineralogias que mais explicavam o aumento da adsorção de nitrato foram a superfície específica, $\mathrm{MO}$ e $\mathrm{pH}$ em $\mathrm{H}_{2} \mathrm{O}$, os quais no presente estudo pode-se observar que o LVd apresenta maior argila e $\mathrm{MO}$ e menor $\mathrm{pH}$ em $\mathrm{H}_{2} \mathrm{O}$ do que o PVAe (Tabelas 1 e 2).

$\mathrm{O}$ íon $\mathrm{NO}_{3}^{-}$é dificilmente retido no solo, sendo sua adsorção principalmente eletrostática, dependente da carga elétrica do solo, o que facilita sua lixiviação e promove perdas desse nutriente do solo, oferecendo ainda risco de contaminação do lençol freático (ALCÂNTARA; CAMARGO, 2005; FEY et al., 2010). Devido o N ser de grande importância na agricultura por ser uma macronutriente essencial ao desenvolvimento de plantas, à décadas a lixiviação do $\mathrm{NO}_{3}^{-}$tem promovido preocupação à saúde pública devido a utilização de fertilizantes nitrogenados (SOLHI et al., 2012). Alguns dos problemas causados devido à lixiviação do $\mathrm{NO}_{3}$ - incluem a eutrofização de corpos de água que podem culminar na morte de peixes e outros organismos, problemas à saúdes de animais tais como bovinos, ovinos e eqüinos que apresentam certas bactérias no trato digestivo que convertem nitrato em nitrito levando-os a uma forma de envenenamento, e à saúde humana principalmente em bebês prejudicando o transporte de oxigênio pelo sangue (RESENDE, 2002).

Conforme a resolução CONAMA n ${ }^{\circ} 357$ de 2005 foi estabelecido que os teores de $\mathrm{NO}_{3}^{-}$em água doce não devem ultrapassar $10 \mathrm{mg} \mathrm{L}^{-1}$ (BRASIL, 2005). Dado o exposto no presente estudo, os resultados encontrados no lixiviado referente aos teores de $\mathrm{NO}_{3}^{-}$, tanto no LVd quanto no PVAe se apresentaram abaixo destes padrões em todos os tratamentos. No entanto, no tratamento com uréia, os teores de $\mathrm{NO}_{3}^{-}$ observados no PVAe (Figura 4) foram próximos aos valores estabelecidos na legislação o que demanda cuidados e monitoramento quanto da aplicação dessa fonte de $\mathrm{N}$ no solo.

Em relação aos teores de $\mathrm{NH}_{4}^{+}$não há padrões específicos estabelecidos de limite desse elemento, podendo este ser mensurado em comparação com os teores máximos de nitrogênio amoniacal total, equivalente a $3,7 \mathrm{mg} \mathrm{L}^{-1}$ para água doce (BRASIL, 2005). Sendo assim, observa-se que os tratamentos com RRB nos dois solos apresentaram teores de $\mathrm{NH}_{4}^{+}$abaixo do estipulado pela legislação (Figura 4). No entanto o tratamento com uréia (U400) no $\mathrm{LVd}$, apresentou um alto teor de $\mathrm{NH}_{4}^{+}$no lixiviado (Figura 4) o qual excede os padrões da legislação estabelecidos para água doce, considerando como referência os padrões de nitrogênio amoniacal total. Este resultado reafirma a demanda de cuidados e monitoramento na aplicação da uréia como fonte de $\mathrm{N}$ no solo por conseqüência do alto potencial de perdas de $\mathrm{N}$ por volatilização da amônia e ainda o risco de contaminação do lençol freático pela lixiviação do $\mathrm{N}$ seja na forma de $\mathrm{NO}_{3}{ }^{-}$ou de $\mathrm{NH}_{4}^{+}$.

\section{Conclusão}

A utilização de RRB no LVd proporcionou efetivo aumento na disponibilidade dos teores de $\mathrm{NO}_{3}{ }^{-}$do solo, sendo, portanto uma fonte de $\mathrm{N}$ ao solo. 
Em relação à lixiviação de $\mathrm{N}, \mathrm{o} \mathrm{LVd}$ apresentou maior capacidade de retenção do ânion $\mathrm{NO}_{3}^{-} \mathrm{e}$ menor do cátion $\mathrm{NH}_{4}^{+}$do que o PVAe.

O lixiviado coletado nos dois solos para os tratamento com RRB apresentou teores de $\mathrm{NO}_{3}^{-}$ e $\mathrm{NH}_{4}^{+}$dentro dos padrões estabelecidos pela legislação durante o período de observações considerado.

O RRB utilizado nesse estudo, em relação à uréia representa menor risco de perdas de $\mathrm{N}$ por volatilização ou lixiviação, com relativamente baixo risco de contaminação de águas subterrâneas, podendo ser, portanto, uma importante fonte alternativa de $\mathrm{N}$ na agricultura.

\section{Agradecimentos}

À Fundação de Amparo à Pesquisa do Estado de Mato Grosso (FAPEMAT) pelo financiamento do projeto e concessão de bolsa de estudo.

\section{Referências}

AGUIAR, M. L.; LOURENÇO, I. P.; OLIVEIRA, T. S.; LACERDA, N. B. Perda de nutrientes por lixiviação em um Argissolo Acinzentado cultivado com meloeiro. Revista Brasileira de Engenharia Agrícola e Ambiental, v. 10, n. 4, p. 811-819, 2006.

ALCÂNTARA, M. A. K.; CAMARGO, O. A. Adsorção de nitrato em solos com cargas variáveis. Pesquisa Agropecuária Brasileira, Brasília, v. 40, n. 4, p. 369-376, 2005.

ANDRADE, I. P.; MONTENEGRO, A. A. A.; SILVA, J. A. A.; FREIRE, M. B. G. S.; SANTOS, T. E. M. Impacto do reúso de efluentes de esgoto no lixiviado de solos cultivados com milho. Revista Brasileira de Engenharia Agricola e Ambiental, Campina Grande, v. 9, p. 212-216, 2005. Suplemento.

ARAÚJO, A. R.; CARVALHO, J. L. N.; GUILHERME, L. R. G.; CURI, N.; MARQUES, J. J. Movimentação de nitrato e amônio em colunas de solo. Ciência Agrotécnica, Lavras, v. 28, n. 3, p. 537-541, 2004.

BETTIOL, W.; FERNANDES, S. A. P. Efeito do lodo de esgoto na comunidade microbiana e atributos químicos do solo. Jaguariúna: Embrapa Meio Ambiente, 2004. 6 p. (Comunicado técnico, 24).
BLACK, A. S.; WARING, S. A. Adsorption of nitrate, chloride and sulphate by some Highly Weathered soils from South-East Queensland. Australian Journal of Soil Research, Melbourne, v. 17, n. 2, p. 271-282, 1979.

BOEIRA, R. C. Lixiviação de nitrogênio em Latossolo incubado com lodo de esgoto. Revista Brasileira de Ciência do Solo, Viçosa, MG, v. 33, n. 4, p. 947-958, 2009.

BOEIRA, R. C.; LIGO, M. A. V.; DYNIA, J. F. Mineralização de nitrogênio em solo tropical tratado com lodo de esgoto. Pesquisa Agropecuária Brasileira, Brasília, v. 37, n. 11, p. 1639-1647. 2002.

BRASIL. Conselho Nacional do Meio Ambiente CONAMA. Resolução $\mathrm{n}^{\circ} 357$, de 17 de março de 2005. Dispõe sobre a classificação dos corpos de água e diretrizes ambientais para o seu enquadramento, bem como estabelece as condições e padrões de lançamento de efluentes, e dá outras providências. Diário Oficial [da] União, República Federativa do Brasil, Poder Executivo, Brasília, DF, n. 53, de 18/03/2005, seção 1. p. 58-63.

Ministério da Agricultura Pecuária e Abastecimento-MAPA. BrasilProjeções do Agronegócio 2011/2012 a 2021-2022. Brasília: Assessoria de Gestão Estratégica, 2012. 50 p. Disponível em: <http://www. agricultura.gov.br/>. Acesso em: 01 dez. 2012.

BREMNER, J. M. Nitrogen-total. In: SPARKS, D. L.; PAGE, A. L.; HELMKE, P. A. et al. Methods of soil analysis. Part 3. Chemical methods. Madison: Soil Science Society of America, 1996. p. 1085-1121.

BREMNER, J. M.; KEENEY, D. R. Steam distillation methods for determination of ammonium, nitrate and nitrite. Analytica Chimica Acta, Oxford, v. 32, p. 482485, 1966.

BRITO, F. L.; ROLIM, M. M.; PEDROSSA, E. M. R. Concentração de cátions presentes no lixiviado de solos tratados com vinhaça. Engenharia Agrícola, Jaboticabal, v. 27, n. 3, p. 773-781, 2007.

CABEZAS, W. A. R. L.; SOUZA, M. A. Volatilização de amônia, lixiviação de nitrogênio e produtividade de milho em resposta à aplicação de misturas de uréia com sulfato de amônio ou com gesso agrícola. Revista Brasileira de Ciência do Solo, Viçosa, MG, v. 32, n. 6, p. 2331-2342, 2008.

CANTARELLA, H. Nitrogênio. In: NOVAIS, R. F.; ALVAREZ V, V. H.; BARROS, N. F.; FONTES, R. L. F.; CANTARUTTI, R. B.; NEVES, J. C. L. Fertilidade do solo. Viçosa: Sociedade Brasileira de Ciência do Solo, 2007. p. 375-470.

CANTARUTTI, R. B.; MARTINS, C. E.; CARVALHO, M. M.; FONSECA, D. M.; ARRUDA, M. E.; VILELA, 
H.; OLIVEIRA, R. T. T. Pastagens. In: RIBEIRO, A. C.; GUIMARÃES, P. T. G.; ALVAREZ V, V. H. (Ed.). Recomendações para o uso de corretivos e fertilizantes em Minas Gerais - $5^{a}$ aproximação. Viçosa: CFSEMG/ UFV, 1999. p. 332-341.

CEMBRANELLI, M.A. R.Lixiviação deíonsinorgânicos em solos que receberam fertilizantes nitrogenados. 2006. Dissertação (Mestrado em Agronomia) - Instituto Agronômico do Curso de Pós-graduação em Agricultura Tropical e Sub-tropical, Campinas.

COELHO, C. F.; RUIZ, H. A.; FERREIRA, P. A.; FRANÇA, G. E.; ARAÚJO, C. A. S.; DUARTE, M. A. Transporte do amônio em colunas com agregados de um Latossolo Vermelho distrófico. Revista Brasileira de Engenharia Agrícola Ambiental, Campina Grande, v. 4, n. 3, p. 362-367, 2000.

DYNIA, J. F.; BOEIRA, R. C.; SOUZA, M. D. Nitrato no perfil de um Latossolo Vermelho ditroférrico cultivado com milho sob aplicações seqüências de lodo de esgoto. In: BETTIOL, W.; CAMARGO, O. Lodo de esgoto: impactos ambientais na agricultura. Jaguariúna: Embrapa Meio Ambiente. 2006. p. 79-89.

EICHLER, V.; SERAPHIN, E. S.; PORTES, T. A.; ROSA, B.; ARAÚJO, L. A.; SANTOS, G. Produção de massa seca, número de perfilhos e área foliar do capimmombaça cultivado em diferentes níveis de nitrogênio e fósforo. Ciência Animal Brasileira, Goiânia, v. 9, n. 3, p. 617-626, 2008.

EMPRESA BRASILEIRA DE PESQUISA AGROPECUÁRIA - EMBRAPA. Manual de métodos de análise de solo. 2. ed. Rio de Janeiro: Centro Nacional de Pesquisas de Solos, 1997. 209 p.

Sistema brasileiro de classificação de solos. 2. ed. Rio de Janeiro: Embrapa Solos, 2006. 312 p.

FERNANDES, F. C. S.; LIBARDI, P. L. Drenagem interna e lixiviação de nitrato em um Latossolo sob sucessão milho-braquiária-milho, com diferentes doses de nitrogênio. Revista Brasileira de Ciência do Solo, Viçosa, MG, v. 33, n. 5, p. 1163-1173, 2009.

FERREIRA, J. C. V. Municípios Mato-Grossenses: pontes e lacerda. In: Mato Grosso e seus municípios. São Paulo: Buriti, 2001. p. 566-568.

FEY, R.; LANA, M. C.; ZOZ, T.; RICHART, A.; LUCHESE, E. B. Relações entre lixiviação de nitrato e produção de biomassa do milho com dejetos suínos provenientes de diferentes tratamentos. Revista Brasileira de Ciências Agrárias, Recife, v. 5, n. 2, p. 212-218, 2010.

FRANCO, J. A. M.; SARAIVA NETO, A. Produção de fertilizantes nitrogenados e suprimento de matériaseca. In: YAMADA, T.; ABDALLA, S. R. S.; VITTI,
G. C. Nitrogênio e enxofre na agricultura brasileira. Piracicaba: IPNI Brasil, 2007. p. 73-108.

FREITAS, G. A.; SANTOS, L. B.; SIEBENEICHLER, S. C.; NASCIMENTO, I. R.; SILVA, R. R.; CAPONE, A. Resíduo de efluente bovino como fertilizante alternativo para a produção de rúcula. Pesquisa Aplicada e Agrotecnologia, Cascavel, v. 3, n. 2, p. 39-44, 2010.

JULIATTI, M. A.; PRADO. R. M.; BARRIQUELO, M. F.; LENZI. E. Cádmio em latossolo vermelho cultivado com milho em colunas: mobilidade e biodisponibilidade. Revista Brasileira de Ciência do Solo, Viçosa, MG, v. 26, n. 4, p. 1075-1081, 2002.

LiSBOA, C. C.; COSTA, S. E. V. G. A.; CASTRO, D. M.; MARQUES, J. J. Efeito da homeopatia ammonium carbonicum na minimização da lixiviação de nitrato. Ciência Agrotécnica, Lavras, v. 31, n. 2, p. 317-325. 2007.

MARTHA JÚNIOR, G. B. M.; VILELA, L.; SOUZA, D. M. G. Adubação nitrogenada. In: Cerrado: uso eficiente de corretivos e fertilizantes em pastagens. Planaltina: Embrapa Cerrados, 2007. p. 117-144.

MARTINES, A. M.; ANDRADE, A. C.; CARDOSO, E. J. B. N. Mineralização do carbono orgânico em solos tratados com lodo de curtume. Pesquisa Agropecuária Brasileira, Brasília, v. 41, n. 7, p. 1149-1155. 2006.

MEKARU, T.; UEHARA, G. Anion adsorption in ferruginous tropical soils. Soil Science Society of American Journal, Madison, v. 36, n. 2, p. 296-300, 1972.

MIYAZAWA, M.; BARBOSA, G. M. C.; PARRA, M. S. Lixiviação de nitrogênio no solo pela aplicação de dejeto de suíno. In: SIMPÓSIO INTERNACIONAL SOBRE GERENCIAMENTO DE RESÍDUOS DE ANIMAIS. USO DOS RESÍDUOS DA PRODUÇÃO ANIMAL COMO FERTILIZANTE, 1., 2009. Anais... Florianópolis: [s.n], 2009. p. 143-147.

MOURALES, M. M.; XAVIER, C. A. M.; SILVA, A. A.; LUCAS JUNIOR, L. Uso da compostagem para tratamento de resíduo sólido de abatedouro de bovinos. Revista Univap, Vale do Paraíba, v. 13, n. 24, p. 136-137, 2006.

OLIVEIRA, F. C.; MATTIAZZO, M. E.; MARCIANO, C. R.; MORAES, S. O. Lixiviação de nitrato em um Latossolo amarelo distrófico tratado com lodo de esgoto e cultivado com cana-de-açúcar. Scientia Agricola, Piracicaba, v. 58, n. 1, p. 171-180, 2001.

OLIVEIRA, J. R. A.; VILELA, L.; YARZA, M. A. Adsorção de nitrato em solos do cerrado do Distrito Federal. Pesquisa Agropecuária Brasileira, Brasília, v. 35, n. 6, p. 1199-1205, 2000. 
PIRES, A. M. M.; MATTIZZO, M. E. Avaliação da viabilidade do uso de residuos na agricultura. Jaguariúna: Embrapa Meio Ambiente, 2008. 9 p. (Circular técnica, 19).

PRIMAVESI, O.; PRIMAVESI, A. C.; CORRÊA, L. A.; SILVA, A. G.; CANTARELLA, H. Lixiviação de nitrato em pastagem de coastcross adubada com nitrogênio. Revista Brasileira de Zootecnia, Viçosa, MG, v. 35, n. 3, p. 683-690, 2006.

RESENDE, A. V. Agricultura e qualidade da água: contaminação da água por nitrato. Planaltina: Embrapa Cerrados, 2002. 28 p. (Documento, 57).

SANTA CATARINA. Manual técnico para coleta de amostras de água. Santa Catarina: Centro de Apoio Operacional do Meio Ambiente - CME, 2008. 37 p. (Coleção Suporte Técnico-Jurídico). Disponível em: $<$ http://www.portal.mp.sc.gov.br>. Acesso em: $01 \mathrm{dez}$. 2012.
SANTOS, S. C. G.; MENEZES, J. F. S.; BENITTES, V. M. Lixiviação de nitrogênio em um Latossolo vermelho cultivado com soja após aplicação de dejetos líquidos de suínos. In: SIMPÓSIO INTERNACIONAL SOBRE GERENCIAMENTO DE RESÍDUOS DE ANIMAIS. USO DOS RESÍDUOS DA PRODUÇÃO ANIMAL COMO FERTILIZANTE, 1., 2009. Anais... Florianópolis: [s.n], 2009. p. 338-344.

SCOTT, A. J.; KNOTT, M. A cluste analysis methods for groping means in the analysis of variants. Biometrics, Raleigh, v. 30, n. 3, p. 507-512, 1974.

SOLHI, S.; SOLHI, M.; SIEF, A.; AGHAKHANI, A.; MOUSAVI, S. F.; ABEDI-KOUPAII, J. Effect of nitrogen fertilizers on nitrate leaching from a saline soil profile under corn and barley cultivation. International Research Journal of Applied and Basic Sciences, Londres, v. 3, n. 3, p. 563-567, 2012.

TEDESCO, M. J.; GIANELLO, C.; BISSANI, C. A.; BOHNEN, H.; VOKWEISS, S. J. Análise de solo, plantas, e outros materiais. 2. ed. Porto Alegre: Universidade Federal do Rio Grande do Sul, 1995. 174 p. (Boletim técnico, 5). 